travel, and hold down a job. Such patients were maintained for long periods on reasonable doses which did not need increasing. Disabling anxiety often returned if the dose was reduced, which is not surprising in patients suffering from those particular chronic illnesses.

Further distress is now occurring in this chronically anxious, and therefore vulnerable, group of patients as a result of the damnation chorus. While the benzodiazepines may be far from ideal, there is still a large population needing treatment for anxiety. This may have to be in the form of medication, even if only because the sheer weight of their numbers makes other forms of treatment unavailable.

R. J. KERRY

21 Whitworth Road

Sheffield S10 3HD

\section{Compulsory HIV testing in psychiatry}

SIR: The Royal College of Psychiatrists' guidelines for the management of HIV-related conditions in psychiatric practice were published recently (Catalan et al, 1989). The British Medical Journal commented that perhaps the most controversial point in the guidelines refers to the legality of testing without consent (British Medical Journal, 1989). The question, however, extends beyond the framework of the current legislation. The 1983 Mental Health Act was drawn up at a time when the HIV test was not yet available and the HIV epidemic was not a major issue on the British agenda. It would be wrong to use such legislation to support controversial clinical procedures which should be primarily driven by ethical, social, and clinical considerations. In the light of this Journal's coverage of these issues to date, I will concentrate on the points which merit further discussion.

1. Placing an emphasis on education and confidentiality when dealing with HIV in psychiatric practice is not an easy option, but is a challenge which could be met with alacrity. It is as well to ask how many psychiatric hospital wards give regular education programmes on AIDS-related risk behaviours to staff and patients, and how many provide ready access to condoms? In the face of staff prejudices, their knowledge of a patient's serostatus can lead to worse rather than better patient care (Cummings et al, 1986).

2. A difficult dilemma might arise in the future when AIDS-related dementia is suspected in a patient, now that treatment with zidovudine has become an experimental option (Schmitt et al, 1988). The evidence at present does not justify compulsory HIV testing and treatment with zidovudine in this condition.
3. I do not believe that HIV testing should be carried out without consent in patients showing aggressive or uninhibited behaviour. From the point of view of the patient, the financial, social, and occupational implications of an HIV test are enormous. Perhaps these need to be further spelt out to proponents of compulsory testing. It has been known in the past, for example, for an insurance company to penalise a candidate for having had an HIV test, whatever the result. From the point of view of staff safety, a negative result could "lull (them) into a false sense of security", as Dunn (1988) has already pointed out. A negative HIV $\mathrm{Ab}$ test can result not only from the sometimes lengthy period to seroconversion, but also from the finite specificity of the tests. It is important, therefore, to ensure staff safety by the provision of adequate numbers of trained staff and protective equipment.

John Hunter Clinic

DANITZa JADRESIC

\section{St Stephen's Clinic}

Fulham Road

London SW10 9TH

\section{Reforences}

British Medical Journal (1989) HIV testing in psychiatry. British Medical Journal, 298, 1600.

Catalan, J., Riccio, M. \& Thompson, C. (1989) HIV disease and psychiatric practice. Psychiatric Bulletin, 13, 316-332.

Cumanngs, M. A., RAPAPORT, M. \& CUMmINGs, K. (1986) A psychiatric staff response to acquired immune deficiency syndrome. American Journal of Psychiatry, 143, 682.

DuNN, J. (1988) Screening for HIV. British Journal of Psychiatry. $153,568-569$.

SCHMTT, F. A., BigleY, J. W., McKinnis, R., et al (1988) Neuropsychological outcome of zidovudine (AZT) treatment of patients with AIDS and AIDS-related complex. New England Journal of Medicine, 319, 1573-1578.

\section{Delusions of pregnancy in men}

SIR: We read with interest Chaturvedi's report (Journal, May 1989, 154, 716-718) of delusions of pregnancy in men. We describe below a patient with delusions of multiple pregnancy and multiple births where, unlike Dr Chaturvedi's case, no obvious organic pathology seemed to account for this symptom.

Case report: A never married, 31 -year-old caucasian male with a 15-year history of chronic undifferentiated schizophrenia was admitted for in-patient evaluation after having failed to respond to adequate trials of three different neuroleptic drugs. He had also developed intolerable symptoms of akathisia and dystonia. All neuroleptic drugs had been discontinued one week prior to admission. Propranolol $(80 \mathrm{mg} /$ day) and lorazepam ( $1.5 \mathrm{mg} /$ day) were discontinued over a period of 7 days after admission. 
On admission, the patient's mental state revealed multiple somatic and grandiose delusions, in addition to thought broadcasting, third person auditory hallucinations (short sentences, mood-incongruent, both conversing and commenting voices), and marked thought disorder. One of his unshakable morbid beliefs was that he had been pregnant ("with four boys and a girl") and that he had delivered quintuplets a year ago in a hospital. He was convinced that while pregnant he could feel pressure on his abdomen and ribs and that "babies were kicking about". He believed that his internal organs had been traded with those of a woman, and hence he had a uterus. He believed that he had impregnated himself. His affect was restricted in its range. He was fully oriented with normal memory.

The patient had been born after $\mathbf{4 2}$ weeks gestation by breech presentation, after prolonged labour ( 48 hours). He developed pneumonia and febrile convulsions at 18 months, which did not recur. His developmental milestones were normal, and he passed all his school grades up to age 16 , when in a period of weeks his grades dropped dramatically, and he developed psychotic symptoms leading to his first psychiatric admission. He has had eight subsequent admissions to psychiatric hospitals. Despite many trials of neuroleptics over the years, his positive symptoms had been refractory to treatment. His family history is notable for psychiatric illness among second-degree relatives (endogenous depression, epilepsy, and schizophrenia). There was no history of drug or alcohol abuse.

Physical and neurological examinations were essentially normal, as were blood biochemistry (including thyroid, liver, and renal profiles), urinalysis, routine haematology, and a computerised tomography scan and EEG. Following treatment with clozapine $(300 \mathrm{mg} /$ day $)$ for 4 weeks, his thoughts were more organised, his hallucinations were less frequent and he was more sociable. However, he remained firm in his belief of having been pregnant and of having delivered quintuplets.

While no gross cerebral pathology was detected, we cannot rule out subtle cerebral dysfunction in view of the history of perinatal complications and febrile convulsions. Like Dr Chaturvedi, we were unable to demonstrate any disturbed family dynamics, a postulated pathogenetic factor in this symptom complex. At a psychopathological level, we wonder whether the preoccupation with bizarre somatic delusions (he believed that his ribs had been "eaten" and replaced, and that organs had been substituted) had led to secondary somatic hallucinations (pressure in his abdomen and ribs, babies moving about), as had been suggested by Hamilton (1976). On the other hand, it may be that somatic hallucinations occurred first and secondarily led to somatic delusions, as it was difficult to get the patient to correctly sequence this symptom, which he had had for approximately 4 months prior to this admission. He displays a retrospective delusion in that while he no longer believes he is pregnant, he backdates his delusion to a year ago, whereas he has held this belief for 4 months.

\section{K. N. R. Chengappa}

S. STEINGARD

J. S. BRAR

M. S. KeshavaN

Schizophrenia Module

Western Psychiatric Institute \& Clinic

3811 O'Hara Street

Pittsburgh, PA 15213-2593

USA

\section{Reference}

Hamilton, M. (1976) Fish's Schizophrenia (2nd edn), pp 45-46. Bristol: John Wright.

SIR: We were most interested to read the paper by Chaturvedi (Journal, May 1989, 154, 716-718). We should like to report a further case.

Case report: The patient was an unmarried 33-year-old caucasian man. He was born prematurely at eight months with Fallot's tetralogy, which was successfully corrected at the age of 11 years. He is of borderline intelligence (WAIS score 70). He attended ESN (M) (educationally subnormalmild) school between the ages of 5 and 15, and then worked as a road sweeper until his first episode of psychosis at the age of 23. It is reported that at that time he heard voices saying that he was a poof, a woman, and that he was masturbating. He was sure that he was pregnant, and was in hospital "to have a vagina put on him".

There was no evidence of clouding of consciousness and he had no insight into his symptoms. He was treated with phenothiazines and ECT, with marked improvement but his symptoms did not completely resolve. Over the next 8 years he continued to receive psychiatric care from general psychiatrists, both as an out-patient and at times as an in-patient, receiving a diagnosis of schizoaffective psychosis.

The patient was admitted recently because he had jumped out of an upstairs window. At interview he expressed his usual paranoid delusions that people were wishing to kill him. He also expressed a firm belief that he was pregnant. When it was pointed out to him that men do not become pregnant, he answered that he knew this but there was always a first time. He was quite concerned about how the baby would be born, as he had no vagina. He thought he had become pregnant by masturbating.

The patient is $5^{\prime} 5 \frac{1}{2}$ tall, obese, with normal genitalia, and has a girlfriend with whom he has a sexual relationship. Investigations showed normal chromosomes and normal biochemistry. His current medication consists of flupenthixol decanoate injections ( $60 \mathrm{mg}$ every three weeks) and chlorpromazine ( $50 \mathrm{mg}$ twice per day).

Dr Chaturvedi's patient was of low intelligence, with an IQ of 42; our patient had an IQ of 70, and unlike Dr Chaturvedi's, did not suffer from epilepsy. 